\title{
An Analysis of Diversity Factors applied to Harmonic Emission Limits for Energy Saving Lamps
}

\author{
V. Ćuk, J.F.G. Cobben, W.L. Kling, and R.B. Timens
}

\begin{abstract}
An experimental investigation of diversity factors for CFLs (compact fluorescent lamps) in combination with LED (light emitting diode) lamps is presented in this paper. Diversity factors were calculated for different configurations of lamps connected to the same feeder in one phase. It was found that the presence of LED lamps with a high apparent power input can lead to considerable damping of harmonics. If the number of CFLs is much greater, this effect was found as very low. Harmonic emission limits for lamps were also analyzed. A conclusion has been made that with a sufficient variety of energy saving lamps phasing out of incandescent lamps should not significantly impact the resultant harmonic distortion.
\end{abstract}

Index Terms--Power quality, Power system harmonics, Attenuation, Lamps, Standards.

\section{INTRODUCTION}

$\mathrm{R}$ ECENT governmental initiatives of a number of countries to ban selling of incandescent light bulbs have brought some attention on the question of the quality of current drawn by energy saving lamps. Energy saving lamps require considerably smaller amounts of active power than incandescent lamps when they have the same luminous flux. On the other hand, due to their non-linear characteristics, energy saving lamps inject harmonic currents into the network. That creates a concern that a very high penetration level of these types of lamps may increase the overall voltage harmonic distortion considerably.

Most literature on this topic is focused on CFLs (compact fluorescent lamps) [1] - [7], due to their wide usage. It is found that most new designs of CFLs with low power rating $(<25 \mathrm{~W})$ have a PF (Power Factor) up to $0.6[1]-[3]$, and

This research has been performed within the framework of the IOP-EMVT research program 'Power Quality and EMC' that is supported financially by SenterNovem. SenterNovem is an agency of the Dutch Ministry of Economic Affairs

V. Cuk is a PhD candidate at the Eindhoven University of Technology, the Netherlands, (e-mail: v.cuk@tue.nl).

J.F.G. Cobben is working at Alliander, the Netherlands. He is also an assistance professor at the Eindhoven University of Technology, the Netherlands, (e-mail: j.f.g.cobben@tue.nl).

W.L.Kling is a full-time professor and the head of the Electrical Energy Systems group at the Eindhoven University of Technology, the Netherlands, (e-mail: w.1.kling@tue.nl).

R.B. Timens is a $\mathrm{PhD}$ candidate at the University of Twente, the Netherlands, (e-mail: R.B.Timens@ewi.utwente.nl). their current THD (total harmonic distortion) is close or above $100 \%$ [1] - [6]. LED (light emitting diode) lamps are not yet addressed on this issue, because they have lower power ratings, and are rarely used for home or office lighting.

When considering a large number of harmonic loads, the resultant harmonic current is not a mere arithmetical sum of individual harmonic currents of loads. There are two diminishing effects influencing the resultant harmonic current of a large group of loads.

The first thing affecting the aggregated current is the attenuation effect. The attenuation effect is defined in the literature as a decrease of the load's current distortion caused by a certain increase of the voltage distortion (it is not applicable for any general configuration of voltage distortions, but it exists in a certain range of harmonic amplitudes and phases). This effect is noticed for CLFs some other types of equipment, and mixtures of equipment. It is described in [4], [5], [7] - [10].

The second thing affecting the summation is the diversity effect. Diversity of topologies in different appliances causes a diversity of phase angles of harmonic currents generated by devices. This leads to a lower vector sum than the arithmetical sum (which neglects phase angles) when different types of loads are present, as described in [8], [9], [11] - [15].

The diversity factor is defined as the ratio between the vector sum (as measured) and the arithmetical sum of harmonics.

Harmonic emission of individual lamps is restricted by the standard IEC 61000-3-2 [16]. Limitations are divided in two categories: lamps with an active input smaller or equal to 25 $\mathrm{W}$ and lamps with an active input exceeding $25 \mathrm{~W}$.

Lamps with active power up to and including $25 \mathrm{~W}$ have a higher margin for emitting harmonics in terms of percents. It is assumed that a larger number of smaller lamps will emit less harmonics than a small number of bigger lamps (due to attenuating effects). However, when examining the absolute currents permitted for both groups (Discussion section), it was noticed that lamps below $25 \mathrm{~W}$ are allowed to emit much higher values of harmonic currents (e.g. with a $P F$ equal to 0.6 , a lamp of $25 \mathrm{~W}$ can emit almost twice as much harmonic current in comparison to a lamp of $50 \mathrm{~W}$ with the same $P F$ ).

This was a reason to further investigate the diversity factors of energy saving lamps without other equipment, using CFLs and LED lamps with an active power under $25 \mathrm{~W}$. A series of 
experiments was conducted, in which the summation of harmonic currents was measured for different numbers of lamps along a low voltage feeder in the laboratory. The addition of LED lamps made it possible to make a restricted generalization of the diversity factors among energy saving lamps. The attenuation effect was not examined in these experiments, all experiment were done with an almost sinusoidal voltage waveform (THD was about $0.25 \%$ ).

When comparing the measured values to arithmetical sums of harmonics (calculated), it was found that the combination of CFLs and LED lamps can lead to relatively high damping effects. In the case when almost only CFLs are present, it was found that reduction due to diversity is limited to small effects (when using a clean sine wave voltage).

The analysis of diversity factors was extended with a theoretical analysis of harmonic emission limits for lamps, to bring some insight into the absolute values of harmonic currents allowed. That brought to a conclusion that present limits are effective enough when there is a sufficient variety of types (operating principles) of lamps used. In another case, if the lighting becomes almost completely made of CFLs, stricter harmonic limits may be needed to limit the voltage distortion.

\section{EXPERIMENTAL METHOD}

Experiments were conducted in the Power Quality laboratory of the Eindhoven University of Technology. Before the experiments with groups of lamps, measurements on single pieces of lamps were done, to obtain characteristics of each type. Powers and the harmonic content of each lamp type were measured according to the instructions from [16]. Some of the type characteristics are presented in Table I. Once the individual current spectrums were known, it was possible to calculate resultant currents of groups of lamps.

TABLE I

MEASURED CHARACTERISTICS OF LAMP TYPES USED
\begin{tabular}{|c|r|r|r|}
\hline Type & P [W] & PF [-] & \multicolumn{1}{|c|}{ THD $_{\mathbf{I}}[\mathbf{\%}]$} \\
\hline CFL 1 & 12.8 & 0.59 & 112.4 \\
\hline CFL 2 & 18.2 & 0.59 & 115.0 \\
\hline CFL 3 & 10.0 & 0.59 & 102.2 \\
\hline CFL 4 & 11.9 & 0.62 & 101.8 \\
\hline CFL 5 & 10.9 & 0.59 & 103.8 \\
\hline CFL 6 & 10.2 & 0.57 & 117.8 \\
\hline LED & 3.3 & 0.05 & 9.3 \\
\hline
\end{tabular}

The experimental setup for experiments with groups of lamps is schematically presented in Fig. 1.

Voltage was generated by a programmable voltage source, California Instruments MX 45, as a one phase clean sine wave voltage of $230 \mathrm{~V}, 50 \mathrm{~Hz}$. Voltage was fed to the loads through a $4 \times 150 \mathrm{~mm}^{2} \mathrm{Al}$ cable with a length of approximately $100 \mathrm{~m}$. At the end of the cable, five radial branches are made at every $20 \mathrm{~m}$. Each branch had a $4 \times 10 \mathrm{~mm}^{2} \mathrm{Cu}$ cable, $20 \mathrm{~m}$ long. This setup is similar to a household distributive feeder in terms of cable lengths and cross-sections. At the end of each branch, a group of six energy saving lamps was connected.
Voltages and currents were measured by an oscilloscope, and later processed in Matlab to obtain their harmonic spectra's, and calculate arithmetic sums of current harmonics. After a measurement was done on several groups of lamps, resultant harmonic currents were compared to values calculated from individual lamp measurements, with phase angles included and without phase angles (arithmetic sum).

Having the measured values of harmonic currents and calculated arithmetic sums, diversity factors for different configurations can be calculated.

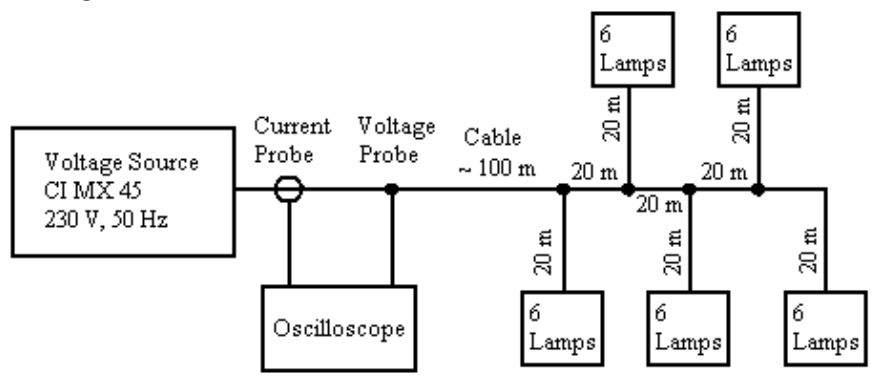

Fig. 1. Schematic diagram of the experiment

In total, 30 lamps were used with seven different types, spread in five groups of six lamps as even as possible. Six types of CFLs with electronic ballasts and one type of LED lamps were used. Different numbers of pieces per type were used. Numbers of pieces used per type are presented in Table II.

TABLE II

NUMBERS OF PEACES USED PER TYPE

\begin{tabular}{|c|c|c|c|c|}
\hline Type & $\begin{array}{c}\text { Number of } \\
\text { peaces }\end{array}$ & Type & Number of peaces \\
\hline CFL 1 & 9 & & CFL 5 & 4 \\
\hline CFL 2 & 3 & & CFL 6 & 2 \\
\hline CFL 3 & 5 & & LED & 2 \\
\hline CFL 4 & 5 & & \multicolumn{2}{|c|}{} \\
\hline
\end{tabular}

Only two LED lamps were used, because presently it is not likely to have a large number of these lamps in comparison to the number of CLFs (mainly due to their lower luminous output). This type of LED lamps has a very low active power input (only $3.3 \mathrm{~W}$ ), but it has a $P F$ equal to 0.05 , resulting in an apparent power of $64.4 \mathrm{VA}$. The number of peaces per CFL type was mainly based on their price. Lamps with a lower price were used in a greater number, based on an assumption that they are used more often than the expensive types.

\section{MEASUREMENT RESULtS}

Several current waveforms of individual lamps are presented in Fig. 2, synchronized to a same phase angle reference. It can be seen that waveforms of CFLs have very similar shapes (scaled by their powers), so it is hard to distinguish the waveforms of CFL 1 and CFL 6 on the picture. The waveform of the LED lamp used has a waveform similar to a capacitor's (because of the phase angle) with some distortion added. 


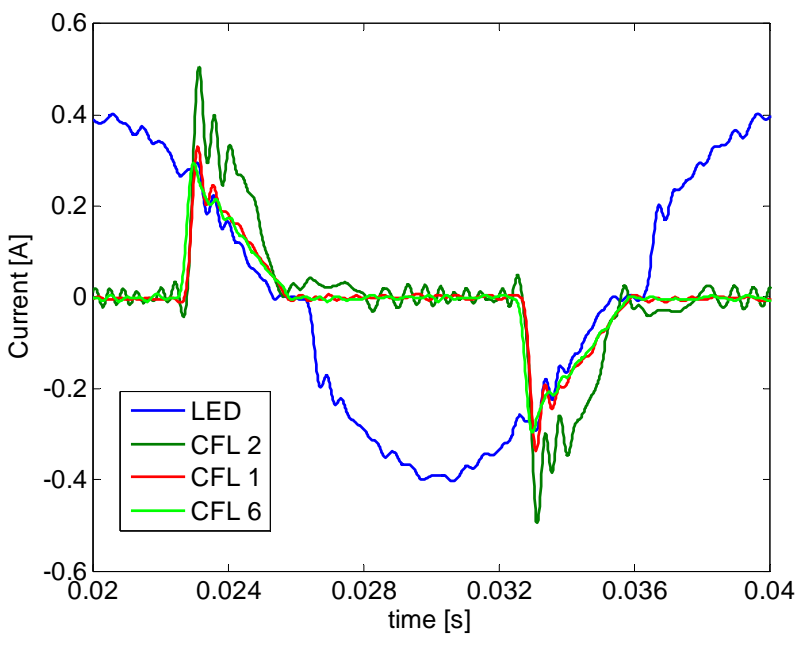

Fig. 2. Current waveforms of several types of lamps used

Relative amplitudes of harmonic currents of all types of lamps are presented in Fig. 3 and their phase angles are given in Fig. 4. Similarities between the waveforms of CFL currents are a consequence of their similar harmonic contents, both in terms of relative harmonic amplitudes and phase angles. Phase angles differ more as the order of harmonics increase, as can be seen in Fig. 4. At certain harmonic orders, the $15^{\text {th }}$ and above the $19^{\text {th }}$, phase angles among CFLs differ significantly. Diversity at these orders does not influence the overall THD considerably, because these orders have low relative amplitudes.
The harmonic content of the LED lamp used is very different, both in terms of amplitudes and phase angles. An important fact is that it has a different phase angle for the $3^{\text {rd }}$ and the $5^{\text {th }}$ harmonic, which have the largest amplitudes among harmonics. Looking at LED's low relative amplitudes (Fig. 3), one may come to a conclusion that it is negligible in comparison to the other lamps used. Still, due to its very low power factor (and relatively high apparent power, see Table I), its harmonic currents are comparable (but still lower than the CFLs).

Measurements with groups of lamps were conducted in the following way: at first, only one group of six lamps was connected at the end of the feeder and the aggregated current was measured; then, measurements were repeated with other groups added, one by one, until all five groups were connected (and all 30 lamps).

Comparisons of measured values and calculated arithmetic sums for 6 lamps are presented in Fig. 5. Diversity factors are presented in Fig. 6. In Fig. 6, diversity factors for even order harmonics are not displayed. These harmonics have very small values, and because of that, calculation is very inaccurate (and also not important). Calculated vector sums are not given in these figures, because they mostly differed only for a few percents from measured values.

It is important to mention that one of the six lamps from the measurement in Fig. 5 and Fig. 6 is a LED lamp. That is the reason for a relatively large difference between the measurement and the arithmetic calculation. In this case the overall current $T H D$ was only $60.3 \%$, because from the total

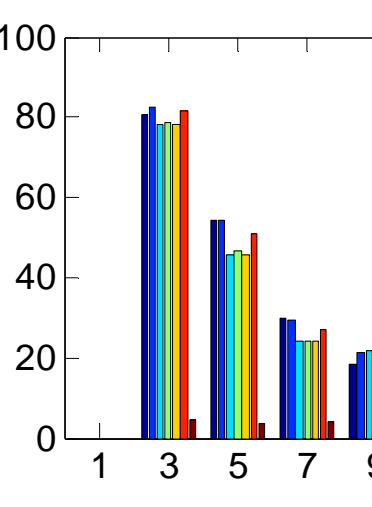

$\begin{array}{lllllllllllllllll}9 & 11 & 13 & 15 & 17 & 19 & 21 & 23 & 25 & 27 & 29 & 31 & 33 & 35 & 37 & 39\end{array}$

Fig. 3. Relative amplitudes of harmonic currents for all types of lamps used
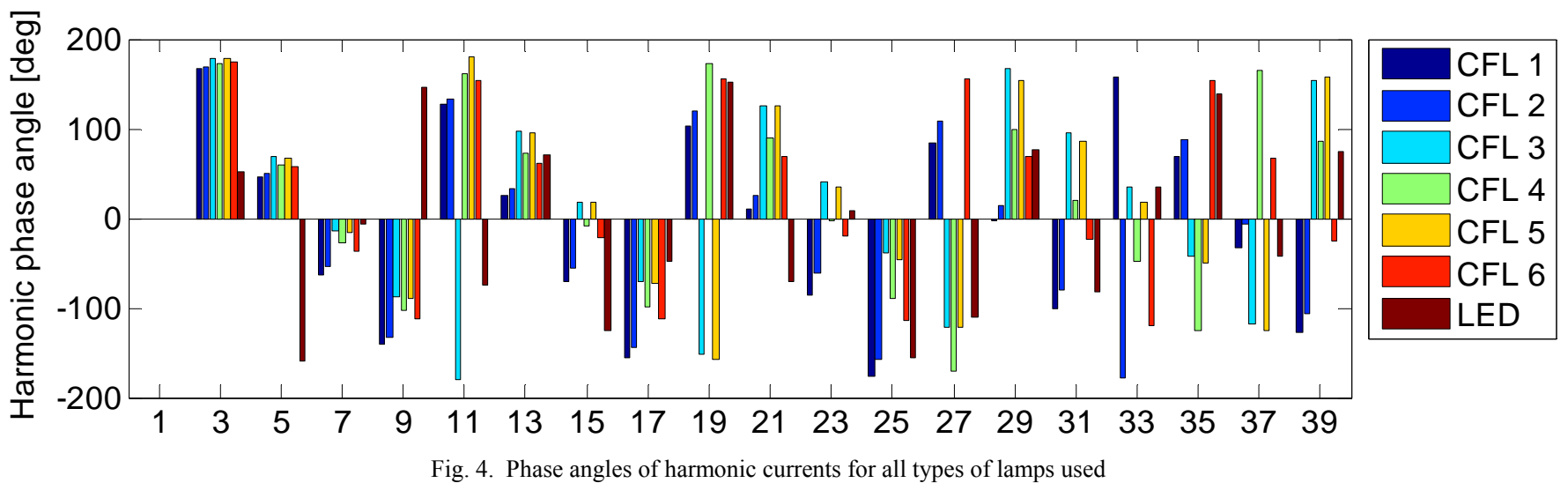

Fig. 4. Phase angles of harmonic currents for all types of lamps used 
148.3 VA the LED part was 64.4 VA (about one third) with a THD of only $9.3 \%$.

Comparisons of measured values and calculated arithmetic sums for 30 lamps are presented in Fig. 7. Diversity factors in this case are presented in Fig. 8. As in Fig. 5, diversity factors for even harmonics are not displayed in Fig. 8.

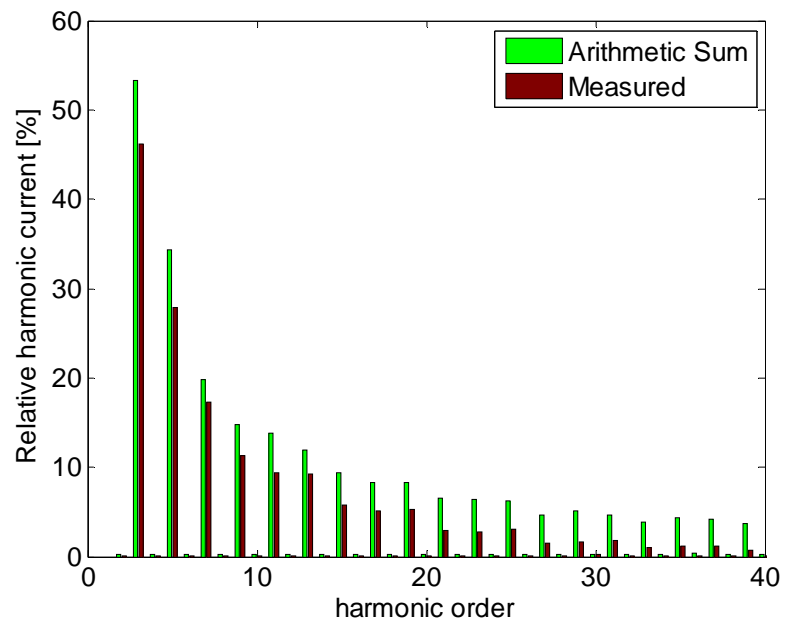

Fig. 5. Comparison of measured harmonics and the arithmetic sum for six lamps

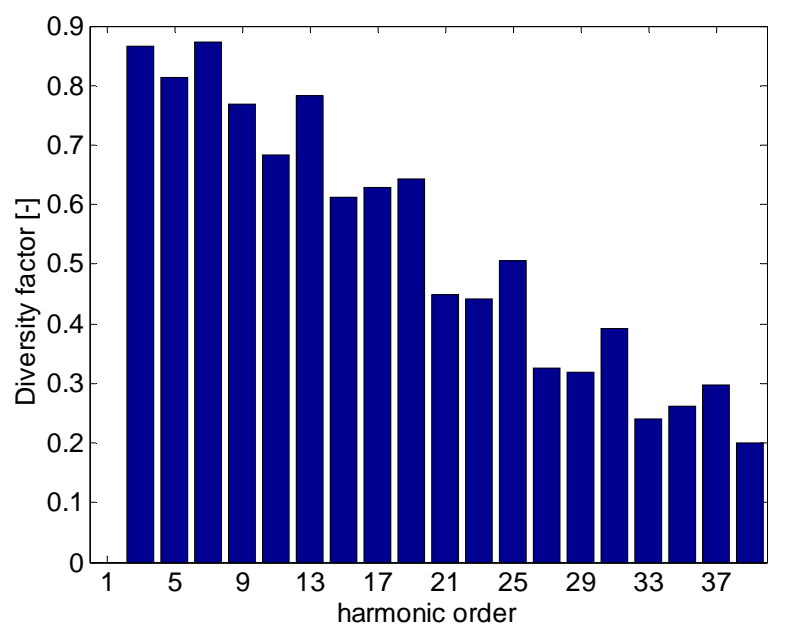

Fig. 6. Diversity factors for different harmonic orders - six lamps

In this case the overall THD was $86.1 \%$ due to the larger content of CFLs (LED lamps represented about one fifth of the apparent power). Also, all diversity factors are higher for 30 lamps (Fig. 6 and Fig. 8) due to the very small diversity among different CFLs. The change of the diversity factor with the number of lamps is further discussed in the Discussion chapter.

\section{DISCUSSION}

\section{A. Diversity factors from measurements}

All devices have a more pronounced diversity on higher harmonic orders, because phase angles of higher frequency components are more sensitive to differences in topology (even for elements that have the same nameplate characteristics).
This property is easily seen on Fig. 6 and Fig. 8. When we look at the situation with only six lamps, all orders above the $25^{\text {th }}$ are reduced from 60 to $80 \%$. With 30 lamps (less LED content) this effect is less visible. The reduction on same orders was from 15 to $30 \%$.

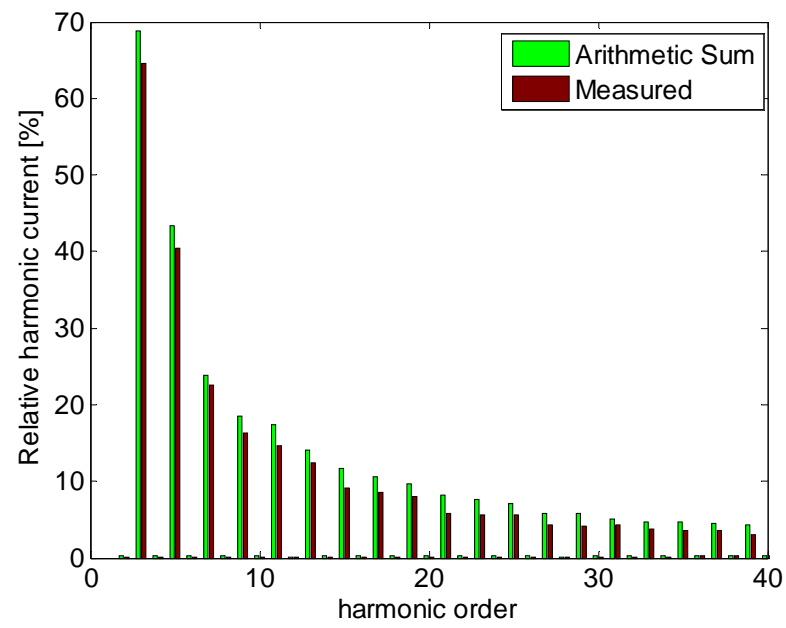

Fig. 7. Comparison of measured harmonics and the arithmetic sum for 30 lamps

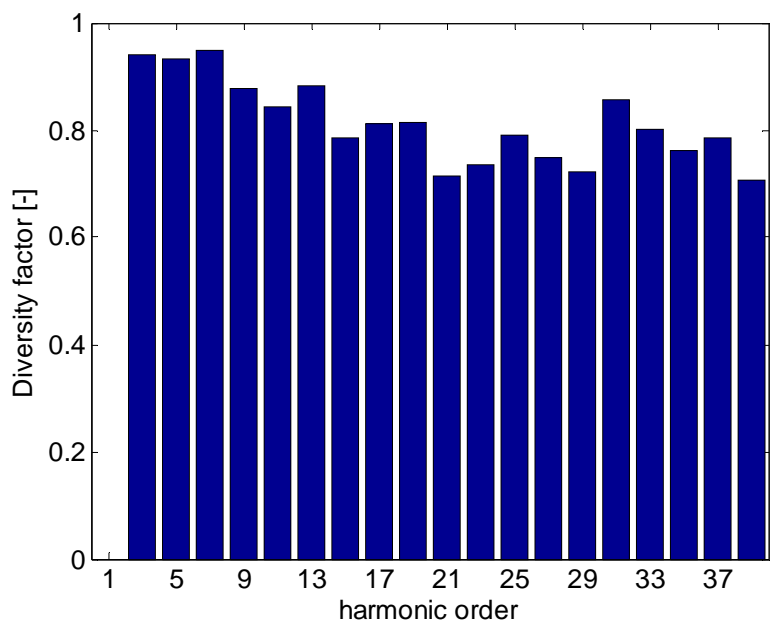

Fig. 8. Diversity factors for different harmonic orders -30 lamps

On low harmonic orders, up to the $11^{\text {th }}$, diversity factors had larger values, with up to $30 \%$ reductions. Also, they were changing less with the addition of more lamps.

Diversity factor changes for several harmonic orders caused by the increase of the number of lamps are shown in Fig. 9. These changes can not be generally used for any configuration of lamps, but they show a clear trend. As the lighting becomes predominantly made of CFLs, even different types of CFLs, reduction due to diversity becomes smaller.

This increase in diversity factors (Fig. 9) is showing a convergence from a case with LED lamps and CFLs to a case with only CFLs. If only CFLs were used, a decrease is expected with a higher number of lamps. The trend would then start from a higher point, and than it would converge towards the same point as this trend, but from the upper side.

It is important to mention that the situation would be different in the presence of voltage distortion and other loads. 
Attenuation due to voltage distortion would probably further reduce the values of harmonic currents. The diversity trend (with the number of lamps) would probably not be changed much, because similar devices react in a similar way to voltage distortion.

Addition of other loads would reduce the values of diversity factors. That is the reason why currents of households do not have a THD close to $100 \%$. The amount of compensation caused by other devices is highly dependent on the timing of their instantaneous operation, but it should be taken into account when emission limits are considered.

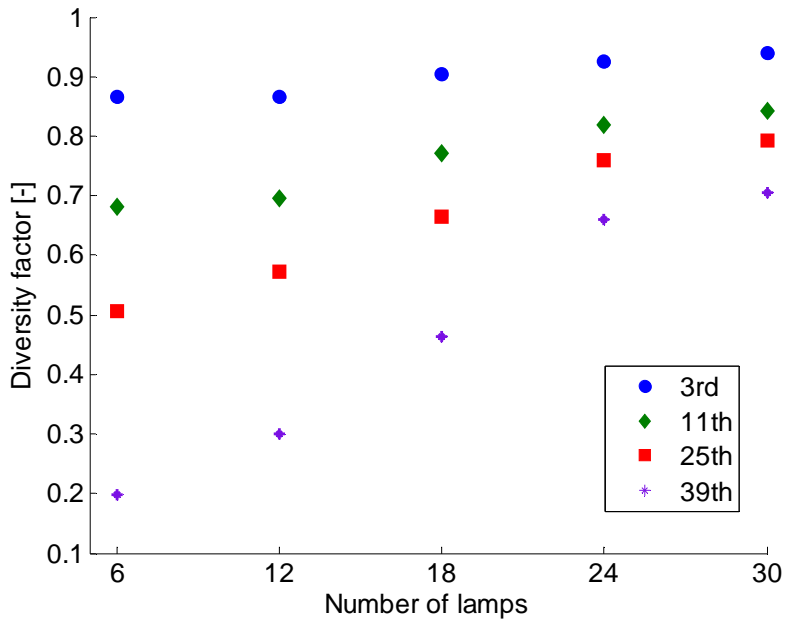

Fig. 9. Changes of diversity factors with the number of lamps

\section{B. Harmonic emission limits for lamps}

As it was mentioned in the introduction, harmonic emission limits for lamps are defined in [16]. They are subdivided into limits for lamps up to the active power of $25 \mathrm{~W}$, and above the active power of $25 \mathrm{~W}$.

Lamps above $25 \mathrm{~W}$ have fixed relative harmonic limits for all orders except the third harmonic (usually the predominant harmonic). The third harmonic has a relative limit related to the $P F$ of the lamp, which means that most of the overall harmonic current is restricted by the lamps apparent power. Using the limits for individual orders, we can calculate the maximal current THD allowed for this power range (maximum is at $P F=1$ ), which has a value of $35 \%$.

Lamps with an active power up to $25 \mathrm{~W}$ have to satisfy at least one out of two criterions.

One of the criteria is that the third harmonic should not exceed $86 \%$ of the fundamental and the fifth harmonic should not exceed $61 \%$. That results in a current THD of approximately $103 \%$ (almost three times higher than in the range above $25 \mathrm{~W}$ ).

The other criterion is given as a table of limits for each harmonic order, where limits are expressed as absolute currents per one watt of active power $(\mathrm{mA} / \mathrm{W})$.

The maximum THD for this range falls into the second criterion and has a very high value (296\%), but it would be applicable only if the $P F$ would have a value of 1 . If the $P F$ has a value of up to 0.75 (and that is usually the case), the first criterion is applicable, and the maximum of $103 \%$.
More important than the relative limits are the absolute limits (in A). Smaller power inputs do not necessarily ask for a proportionally higher number of units. A comparison of allowed absolute harmonic currents in a range from 0 to $50 \mathrm{~W}$ is presented in Fig. 10, as a function of active power and $P F$. Allowed currents are calculated from individual harmonic components as

$$
I_{a b s h}=\sqrt{\sum_{n=2}^{40} I_{n}^{2}}
$$

where $I_{a b s h}$ is the absolute harmonic current, and $I_{n}$ are harmonic components of order $n$.

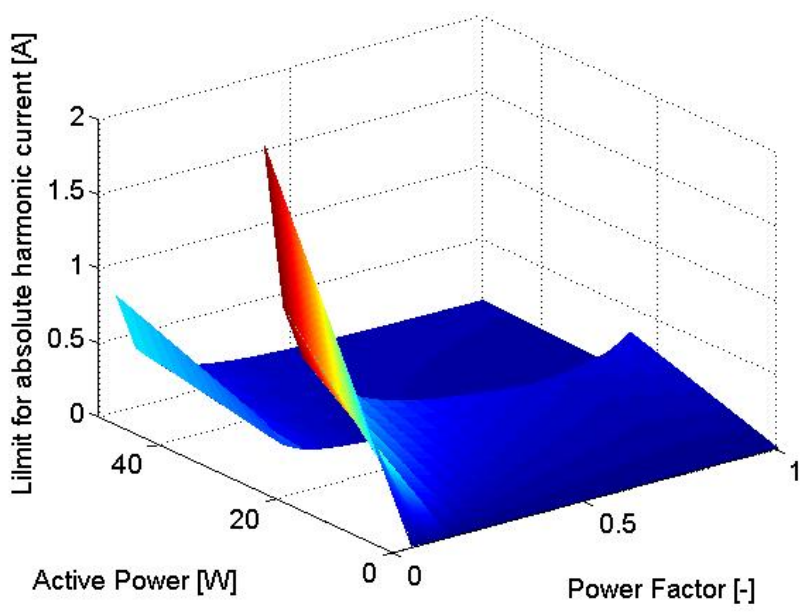

Fig. 10. Absolute harmonic current limits for lamps up to $50 \mathrm{~W}$

Limits are higher in the range up to $25 \mathrm{~W}$, even in terms of absolute currents. The big peak at power factors close to 0 (part of the surface that is red) is not of big concern, because it is not likely that a lamp has such a $P F$ and distortion at the same time.

All CFLs used had a PF between 0.57 and 0.62 , which makes it interesting to look at the limits from Fig. 10 only for $P F=0.6$. This is displayed in Fig. 11, together with measured values of $P$ and $I_{a b s h}$ for all CFLs used.

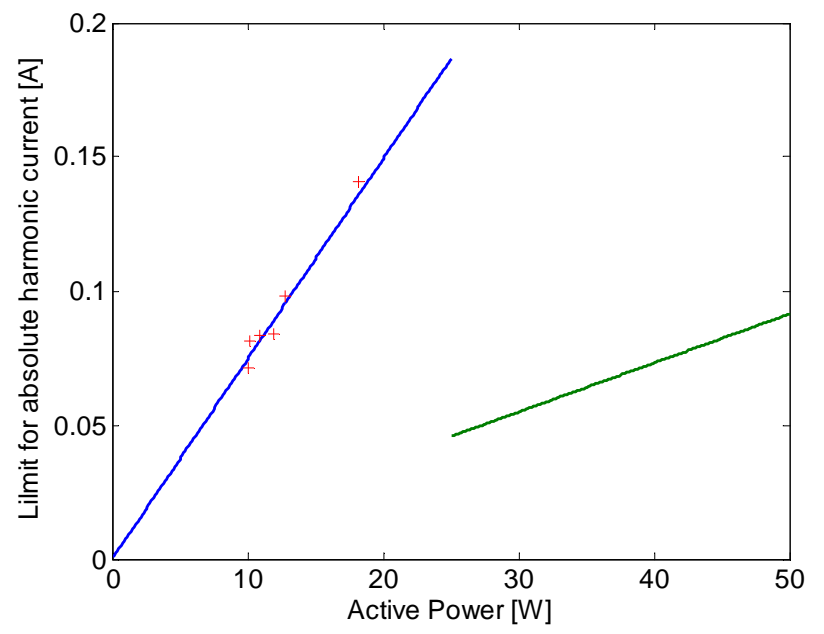

Fig. 11. Harmonic current limits for $\mathrm{PF}=0.6$ and lamp measurements 
As can be seen on Fig. 11, all CFLs used in the experiments are just below or just above the limits. If we consider the diversity factors measured in the laboratory, it can be concluded that only a big variety of energy saving lamps can achieve considerable harmonic reductions among them. If that variety is not available, reduction will depend only on other devices, to compensate for their currents with a THD around $100 \%$.

This does not necessarily mean that stricter rules have to be applied to energy saving lamps because incandescent lamps are being phased out, but if voltage distortions start increasing, that would be one way to influence it.

\section{CONCLUSIONS}

An analysis of diversity factors for energy saving lamps is presented in this paper. Several configurations of CFLs and LED lamps were connected to a radial low voltage feeder and measurements of harmonic currents and diversity factors were conducted.

Measurements showed that a mixture of LED lamps and CFLs can considerably decrease the amount of higher order harmonics. On the other hand, the lower order harmonics did not exhibit a very large reduction.

Harmonic emission limits were also analyzed, and allowed amounts of harmonic currents in both active power ranges were calculated. It was concluded that every time a lamp from a range above $25 \mathrm{~W}$ is substituted by a lamp with up to $25 \mathrm{~W}$, harmonic current is significantly increased.

Phasing out of incandescent lamps will definitely lead to an increase of harmonics in networks. If almost only CFLs are used in that process, it may require stricter harmonic emission limits for lamps. If a bigger mixture of lamp types is used in the process, the harmonic distortion impact will be reduced. The overall diversity factor of a group of customers separated by cables will lead to further reduction of harmonic distortion.

\section{ACKNOWLEDGMENT}

This research has been performed within the framework of the IOP-EMVT research program 'Power Quality and EMC' that is supported financially by SenterNovem. SenterNovem is an agency of the Dutch Ministry of Economic Affairs.

\section{REFERENCES}

[1] R. R. Verderber, O. C. Morse, and W. R. Alling, "Harmonics from compact fluorescent lamps", IEEE Trans. Industry Applications, vol. 29, no. 3, May/June 1993.

[2] R. Arsenau and M. Ouellette, "The effects of supply harmonics on the performance of compact fluorescent lamps", IEEE Trans. Power Delivery, vol. 8, no. 2, April 1993.

[3] J. Cunill-Solà and M. Salichs, "Study and characterization of waveforms from low-watt $(<25 \mathrm{~W})$ compact fluorescent lamps with electronic ballasts", IEEE Trans. Power Delivery, vol. 22, no. 4, October 2007.

[4] A. B. Nassif and J. Acharya, "An investigation on the harmonic attenuation effect of modern compact fluorescent lamps", presented at the $13^{\text {th }}$ International Conference on Harmonics and Quality of Power, Wollongong, Australia, 2008.

[5] A. B. Nassif and W. Xu, "Characterizing the harmonic attenuation effect of compact fluorescent lamps", IEEE Trans. Power Delivery, vol. 24, no. 3, July 2009.
[6] P. N. Korovesis, G. A. Vokas, I. F. Gonos, and F. V. Topalis, "Influence of large-scale installation of energy saving lamps on the line voltage distortion of a weak network supplied by a photovoltaic station", IEEE Trans. Power Delivery, vol. 19, no. 4, October 2004.

[7] N. R. Watson, T. L. Scott, and S. J. J. Hirsch, "Implications for distribution networks of high penetration of compact fluorescent lamps", IEEE Trans. Power Delivery, vol. 24, no. 3, July 2009.

[8] A. Mansoor, W. M. Grady, A. H. Chowdhury, and M. J. Samotyj, "An investigation on harmonics attenuation and diversity among distributed single-phase power electronic loads", IEEE Trans. Power Delivery, vol. 10, no. 1, January 1995.

[9] A. Mansoor and W. M. Grady, "Analysis of compensation factors influencing the net harmonic current produced by single-phase nonlinear loads", presented at the $8^{\text {th }}$ International Conference on Harmonics and Quality of Power, Athens, Greece, 1998.

[10] M. Raylander, W. M. Grady, and M. Narendorf Jr., "Experimental apparatus, testing results, and interpretation of the impact of voltage distortion on the current distortion of typical single-phase loads", IEEE Trans. Power Delivery, vol. 24, no. 2, April 2009.

[11] M. T. Au and J. Milanović, "Establishing harmonic distortion level of distribution network based on stochastic aggregate harmonic load models", IEEE Trans. Power Delivery, vol. 22, no. 2, April 2007.

[12] S. Hansen, P. Nielsen, and F. Blabjerg, "Harmonic cancellation by mixing nonlinear single-phase and three-phase loads", IEEE Trans. Industry Applications, vol. 36, no. 1, January/February 2000.

[13] Edited by P.F. Ribeiro, "Time - varying waveform distortions in power systems", John Wiley \& Sons, 2009.

[14] P. F. Ribeiro, "Investigations of harmonic penetration in transmission systems", chapter 6, PhD Thesis, The Victoria University of Manchester, UK, 1985.

[15] Probabilistic aspects task force of the harmonics working group, "Timevarying harmonics: Part II - Harmonic summation and propagation", IEEE Trans. Power Delivery, vol. 17, no. 1, January 2002.

[16] Electromagnetic Compatibility (EMC) - Part 3-2: Limits - Limitation of voltage changes, voltage fluctuations and flicker in public low-voltage supply systems, for equipment with rated current $\leq 16 \mathrm{~A}$ per phase and not subject to conditional connection, IEC standard 61000-3-2, Ed. 3, November 2005. 